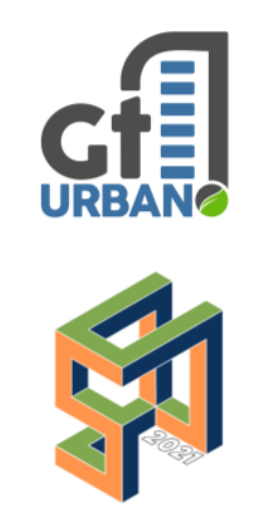

SINGEURB

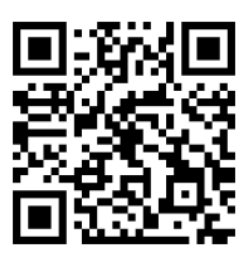

Como citar:

CAMPOS, Larissa S.; MARINHO, Higor

P.; BALTAR, Marina

L. de B.; CORDEIRO,

Carol C. M.;

BENDER, Juliane

Érika C. Avaliação

dos padrões de

viagens realizadas

para um polo

gerador de viagem:

Estudo de caso no

campus Cuiabá da

Universidade Federal

de Mato Grosso. In:

III SIMPÓSIO

NACIONAL DE

GESTÃO E

ENGENHARIA

URBANA:

SINGEURB, 2021,

Maceió. Anais...

Porto Alegre:

ANTAC, 2021. p. 455-

462.

Disponível em:

https://eventos.antac. org.br/index.php/sin geurb/issue/view/14

\title{
Avaliação dos padrões de viagens realizadas para um polo gerador de viagem: Estudo de caso no campus Cuiabá da Universidade Federal de Mato Grosso
}

\section{Evaluation of travel patterns to a travel generator pole: Case study in the Universidade Federal de Mato Grosso, Cuiabá campus}

Larissa Silva Campos, Universidade Federal de Mato Grosso, slarissacampos@gmail.com

Higor Peres Marinho, Universidade Federal de Mato Grosso, higorpmarinho@gmail.com

Marina Leite de Barros Baltar, Universidade Federal de Mato Grosso, mabaltar@gmail.com

Carol Cardoso Moura Cordeiro, Universidade Federal de Mato Grosso, carolcardoso.eng@gmail.com

Juliane Érika Cavalcante Bender, Centro Federal de Educação Tecnológica de Minas Gerais, juliane.erika@gmail.com

\section{RESUMO}

As Instituições de Ensino Superior são Polos Geradores de Viagens e a existência desse tipo de edificação, atrai uma grande quantidade de viagens, pelos diferentes modos, influenciando na mobilidade urbana. Este artigo busca analisar características socioeconômicas dos usuários do campus da Universidade Federal de Mato (UFMT) em Cuiabá. Foram utilizadas as informações provenientes de um projeto de pesquisa sobre a mobilidade do campus universitário, que realizou uma pesquisa de origem-destino na comunidade acadêmica. Foi desenvolvido um zoneamento urbano e construída matriz origem-destino para conhecer o perfil do usuário e caracterizar as viagens de acesso a instituição, explorando as suas diferenças espaciais e socioeconômicos, como também verificando como isso influencia na escolha do modo de transporte. O transporte individual motorizado é o mais utilizado, com total de $43,36 \%$, sendo mais representativo em zonas centrais e de renda alta e média. Além disso, constatou-se que as viagens através de ônibus somam 36,34\% do total, sendo característica de regiões periféricas e de renda média mais baixa. Desta forma, nota-se que a renda familiar, distância média percorrida e o uso e ocupação do solo influenciam na escolha do modo de transporte utilizado.

Palavras-chave: Transporte individual motorizado, Zoneamento, Escolha modal.

\section{ABSTRACT}

Higher Education Institutions are considered Travel Generator Poles since the existence of this type of facilities attracts a large amount of travel, through different modes, affecting urban 
mobility. This article analyzes the socioeconomic characteristics of Universidade Federal de Mato Grasso (UFMT) in Cuiabá campus' users. Information from a research project on the mobility of the university campus, which carried out an origin-destination survey in the academic community, was used. An urban zoning was developed and an origin-destination matrix was built to characterize its user and the access trips to the institution, exploring their spatial and socioeconomic differences, as well as verifying how this influences the choice of transport mode. Private transport is the most used, with a total of $43.36 \%$, being more representative in central and high-and middle-income areas. Furthermore, it was found that trips by bus account for $36.34 \%$ of the total, being characteristic of peripheral regions and of lower middle income. Thus, it is noted that family income, average travel distance and land use and occupation can influence the mode choice.

Keywords: Private transportation, Zoning, Mode choice.

\section{INTRODUÇÃO}

Para a REDEPGV (2019), as Instituições de Ensino Superior (IES) são consideradas um tipo especial de Polos Geradores de Viagens (PGV) devido ao seu potencial de atrair e produzir viagens. Estas são realizadas, em sua totalidade, pelos alunos, prestadores de serviços, servidores e visitantes da instituição de ensino que podem se deslocar por diferentes modos de transportes. Estudos apontam que nas IES a maioria das viagens é realizada por automóveis e, devido ao horário de início e término das atividades letivas coincidirem com o horário de pico da cidade, causam um impacto negativo no tráfego da região (SOUZA, 2007).

Em Cuiabá-MT está instalada a Universidade Federal de Mato Grosso (UFMT) e provisoriamente o campus universitário de Várzea Grande - até a inauguração do novo campus. Baltar (2010) aponta que, no ano de 2010, cerca de 8.500 veículos por dia trafegavam nos dois sentidos do campus universitário, transportando cerca de 15.000 usuários.

Nesse contexto, o objetivo desse artigo é apresentar e caracterizar os padrões de viagens com destino ao campus de Cuiabá da UFMT explorando as suas diferenças espaciais, socioeconômicos e no uso dos diferentes modos de transporte. O estudo visa contribuir para a caracterização das escolhas de transporte por parte dos usuários do campus buscando entender fatores que podem influenciar nessa decisão e possibilitar a criação de políticas que busquem gerenciar a demanda existente.

\section{INSTITUIÇÕES DE ENSINO SUPERIOR COMO POLOS GERADORES DE VIAGEM}

Os PGVs são instalações de naturezas distintas que desenvolvem atividades de porte e escala capaz de produzir um contingente significativo de viagens (PORTUGAL; GOLDNER, 2003). IES apresentam características específicas que requerem cuidados especiais na análise do impacto que produzem sobre a circulação do tráfego nas vias próximas (ALVES, 2015). Ao estudá-las devem ser considerados todos os fatores que alteram a circulação local, como o perfil dos usuários que utilizam o espaço, o modo de deslocamento, classificação econômica, entre outros.

Tendo em vista a problemática apresentada, a UFMT campus Cuiabá já foi objeto de estudo de alguns trabalhos: Medeiros et al. (2019) que analisaram as viagens realizadas por transporte ativo no campus, Belufi et al. (2019) que avaliaram o padrão de adequação dos modos de transporte utilizados para acessar o campus, e Bender e Baltar (2019) que caracterizaram os deslocamentos realizados pelos usuários do local. 


\section{MATERIAIS E MÉTODO}

\subsection{Caracterização do campus universitário}

A população usuária da universidade do campus Cuiabá, em 2019, totalizava 14.847 pessoas, sendo distribuídas em: 1.245 docentes, 1.007 técnicos-administrativos, 11.393 alunos de graduação e 1.192 alunos de pós-graduação (UFMT, 2019). O referido campus é apresentado na Figura 1.

Figura 1 - Localização do campus universitário da UFMT

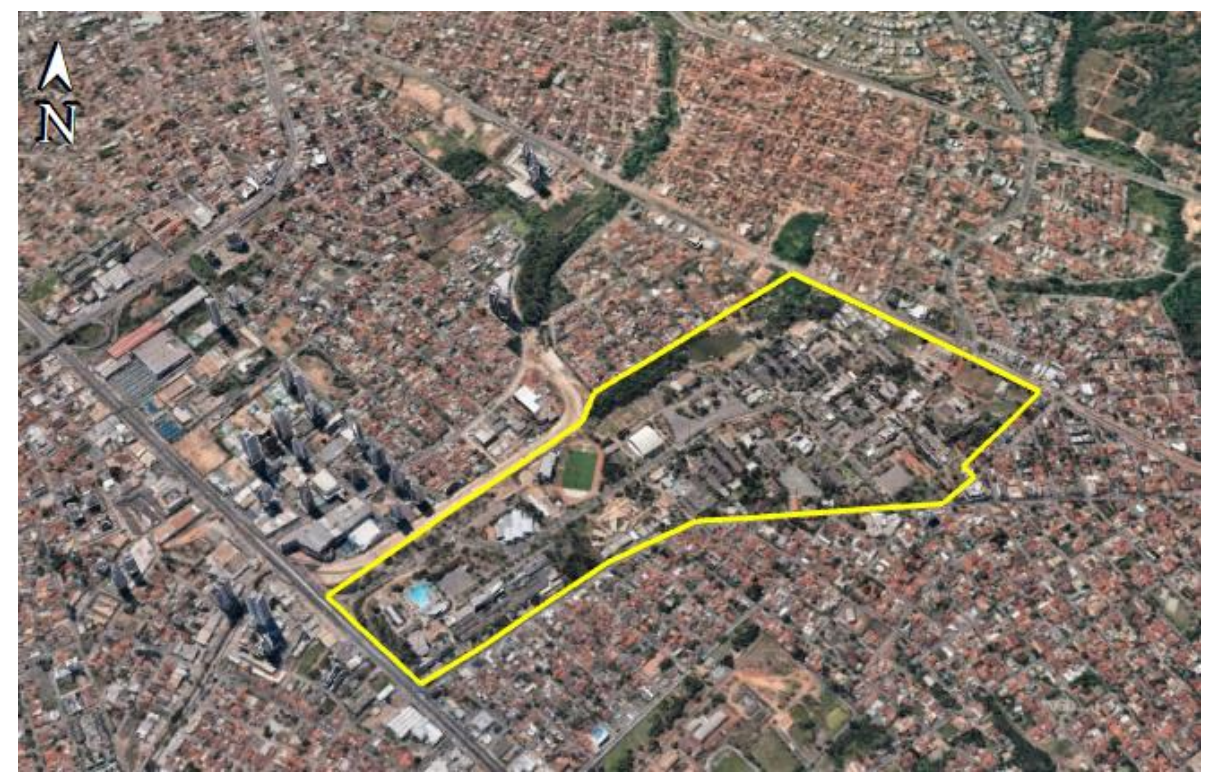

Fonte: Google Earth (2020)

\subsection{Base de dados}

O banco de dados empregado é resultante de uma pesquisa realizada com a comunidade acadêmica do campus por meio de um questionário on-line desenvolvido e aplicado por Bender e Baltar (2019). A pesquisa foi divulgada via e-mail institucional e mídias sociais ao longo dos meses de fevereiro e março de 2019. Foram utilizados os seguintes parâmetros disponíveis no banco de dados: vínculo com a UFMT, município e bairro de origem, modo de transporte utilizado, perfil socioeconômico, município e bairro do trabalho, e destinos para UFMT. Quanto à divisão do modo de transporte foram consideradas sete opções: a pé, bicicleta, carro, motocicleta e transporte público (ônibus), carona e aplicativo (app).

O controle amostral realizado consistiu na verificação dos resultados incompletos ou errôneos, por falta ou inconsistência da informação ou por se tratar de outros campi da UFMT em cidades diferentes, resultando em uma redução de $2 \%$ da amostra. Diante disso foram utilizadas, ao todo, 1.374 respostas, correspondendo a aproximadamente $9 \%$ da população, com um nível de confiança de $95 \%$ e erro amostral de $3 \%$. Considerase, dessa forma, que a amostra obtida é representativa dos usuários do campus. 


\subsection{Zoneamento urbano de Cuiabá e recursos computacionais}

Para o zoneamento urbano da cidade considerou-se, inicialmente, o abairramento de Cuiabá realizada pela prefeitura no ano de 2005. Neste documento verifica-se a divisão dos bairros do município em quatro regiões, sendo elas a região oeste, norte, leste e sul. A partir desse cenário, realizou-se divisão das regiões em zonas de tráfego conforme a semelhança de suas características socioeconômicas como também mediante a presença dos divisores físicos, como as avenidas principais da cidade.

Foi adotado o software Qgis (QGIS, 2021) para realizar o gerenciamento de dados, sinalizar e quantificar as distâncias de origem e destino do zoneamento urbano. A partir dessas informações, efetuou-se o zoneamento da cidade agrupando bairros com renda familiar média semelhante.

\section{RESULTADOS E DISCUSSÕES}

Foram criadas 17 zonas para a cidade de Cuiabá, a Figura 2 ilustra a organização espacial do zoneamento.

Observa-se que a soma de quatro zonas com maior volume de viagens, O1, L2, L3 e L4, corresponde a um valor de $60,04 \%$ do total das 1.374 das respostas analisadas. Essas zonas são caracterizadas pela presença de condomínios residenciais e prédios comerciais, o que influencia no volume de viagens oriundas dessa região. Por outro lado, as zonas O3, S1, S4 e S6, geram apenas 5,31\% do total, denotando uma maior influência da UFMT em bairros mais próximos.

Também é verificado por meio da pesquisa que a maioria dos deslocamentos é realizado por transporte individual motorizado, o que corresponde a aproximadamente $43,48 \%$ do total da amostra. Em contrapartida, o transporte público representa $36,34 \%$ das viagens realizadas, seguido pela modalidade a pé com $14,92 \%$ e os demais modos somando $5,26 \%$. Os dados evidenciam a baixa participação do transporte ativo e corroboram com as conclusões de Medeiros et al. (2019), apontando o predomínio do transporte motorizado em detrimento a outros meios de transportes.

Partindo para uma análise em relação a renda, o modo de transporte e a distância de deslocamento até a UFMT, ao analisarmos as zonas com renda familiar média de $\mathrm{R} \$ 5.000$ a $\mathrm{R} \$ 10.000$, verifica-se que o modo motorizado individual é o mais utilizado, com um percentual médio de $56,41 \%$, enquanto a porcentagem para o transporte público está em torno de 35,34\%, como indicado na Tabela 1. Observa-se também que pelo zoneamento urbano realizado apenas duas zonas se enquadram nessa renda, correspondendo a $12 \%$ da amostra (Vide Figura 2). Além disso, há um aumento do percentual de uso de transporte coletivo com o aumento da distância. 
Figura 2 - Zoneamento de Cuiabá

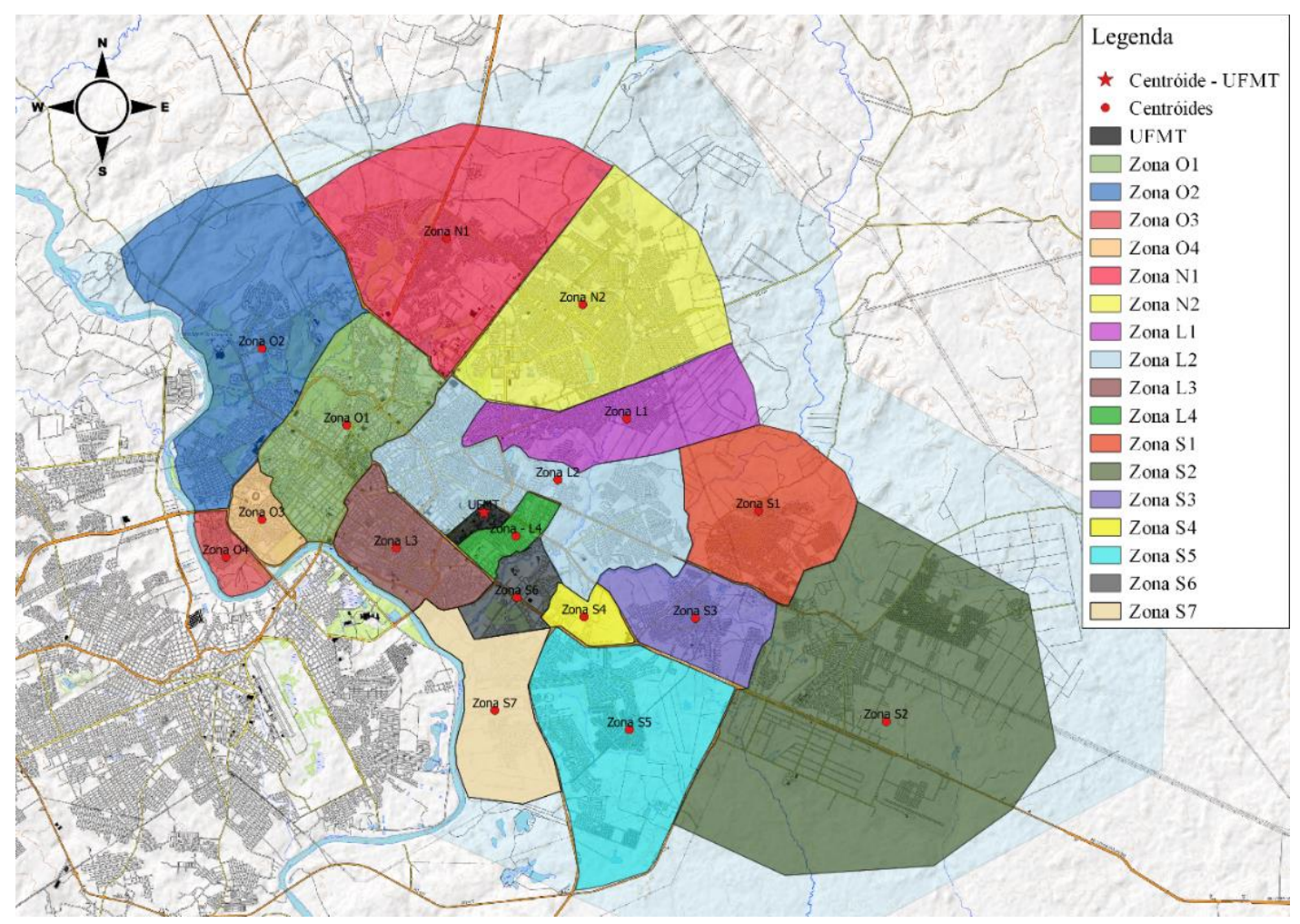

Fonte: Os autores

Tabela 1 - Distribuição quantitativa do modo motorizado individual e público para renda de 5 a 10 mil reais

\begin{tabular}{c|c|c|c|c}
\hline \multirow{2}{*}{$\begin{array}{c}\text { Renda } \\
\text { De R\$ 5.000 a } \\
\text { R\$ } 10.000\end{array}$} & O1 & Transporte individual (\%) & $\begin{array}{c}\text { Transporte público } \\
(\%)\end{array}$ & $\begin{array}{c}\text { Distância } \\
(\mathrm{km})\end{array}$ \\
\cline { 2 - 5 } & O2 & 59,20 & 31,20 & 4,37 \\
\hline Média & - & 53,62 & 43,48 & 7,42 \\
\hline Desvio padrão & - & 56,41 & 35,34 & 5,89 \\
\hline
\end{tabular}

Fonte: Os autores

Todavia, no agrupamento com renda média de $\mathrm{R} \$ 3.000$ a $\mathrm{R} \$ 5.000$, que engloba uma maior quantidade de zonas, nota-se que as três zonas mais distantes são as com maior percentual do transporte público. Entretanto, de forma geral, há uma predominância do transporte individual em relação ao transporte coletivo, com percentuais de $47,13 \%$ e 35,25\% respectivamente, de acordo indicado na Tabela 2.

O último agrupamento é o que apresenta a menor renda média e o que possui a maior porcentagem de utilização de ônibus, somando um total de $62,09 \%$. Por sua vez, o transporte individual tem representatividade de 35,29\%, conforme a Tabela 3. Nesse caso também é verificado o aumento do percentual do transporte público com a distância. 
É observada uma relação entre renda e o uso do transporte individual, visto que o número de deslocamentos através desse modo é realizado por indivíduos com a maior renda familiar média. Araújo et al. (2011) afirma que esse cenário é decorrente de fatores externos, como a estrutura física da cidade, a disposição física das construções, áreas de uso público e a oferta de meios de transportes, influenciando na decisão de adoção de transportes individuais.

Todavia, a falta de integração dos modos de transporte e o não planejamento de sua utilização, como ferramenta de mudanças e de desenvolvimento urbano no munícipio, refletem as disparidades entre as zonas, principalmente, aquelas localizadas em regiões periféricas (uma distância média de 6,88 $\mathrm{km}$ até a instituição) com renda de até $\mathrm{R} \$ 3.000$.

Tabela 2 - Distribuição quantitativa do transporte individual e transporte público para renda de 3 a 5 mil reais

\begin{tabular}{c|c|c|c|c}
\hline \multirow{2}{*}{ Renda } & Zonas & Transporte individual (\%) & $\begin{array}{c}\text { Transporte público } \\
(\%)\end{array}$ & $\begin{array}{c}\text { Distâncias } \\
(\mathrm{km})\end{array}$ \\
\hline \multirow{4}{*}{$\begin{array}{c}\text { De R\$3.000 a } \\
\text { R\$5.000 }\end{array}$} & O3 & 47,62 & 52,38 & 5,98 \\
\cline { 2 - 5 } & O4 & 50,00 & 47,92 & 7,05 \\
\cline { 2 - 5 } & L3 & 59,79 & 27,40 & 1,99 \\
\cline { 2 - 5 } & S6 & 42,76 & 23,03 & 2,55 \\
\cline { 2 - 5 } & S7 & 25,49 & 8,63 & 1,07 \\
\hline Média & - & 58,33 & 33,33 & 2,46 \\
\hline Desvio padrão & - & 45,95 & 54,05 & 5,32 \\
\hline
\end{tabular}

Fonte: Os autores

Tabela 3 - Distribuição quantitativa do transporte individual e transporte público para renda de até 3 mil reais

\begin{tabular}{c|c|c|c|c}
\hline \multirow{2}{*}{ Renda } & Zonas & Transporte individual (\%) & $\begin{array}{c}\text { Transporte público } \\
(\%)\end{array}$ & $\begin{array}{c}\text { Distâncias } \\
(\mathrm{km})\end{array}$ \\
\hline \multirow{4}{*}{ Até R\$3.000 } & $\mathrm{L} 1$ & 28,00 & 70,00 & 4,59 \\
\cline { 2 - 5 } & $\mathrm{N} 1$ & 33,33 & 65,79 & 7,42 \\
\cline { 2 - 5 } & $\mathrm{N} 2$ & 44,00 & 54,00 & 6,18 \\
\cline { 2 - 5 } & $\mathrm{S} 1$ & 36,84 & 63,16 & 7,39 \\
\cline { 2 - 5 } & $\mathrm{S} 2$ & 25,00 & 69,44 & 12,19 \\
\cline { 2 - 5 } & $\mathrm{S} 3$ & 41,46 & 58,54 & 6,36 \\
\cline { 2 - 5 } & $\mathrm{S} 4$ & 34,78 & 56,52 & 7,03 \\
\cline { 2 - 5 } & $\mathrm{S} 5$ & 38,89 & 59,26 & 6,88 \\
\hline Média & - & 35,29 & 62,09 & 2,34 \\
\hline Desvio padrão & - & 6,05 & 5,57 & \\
\hline
\end{tabular}

Fonte: Os autores

É importante ressaltar que a periferização da população é uma característica do espraiamento urbano, fenômeno responsável pelo crescimento urbano desconcentrado (NADALIN; IGLIORI, 2015). Para 
Gomides (2006), o uso ampliado do modo motorizado individual favorece a dispersão das atividades, dificultando a acessibilidade urbana para aqueles que dependem unicamente do transporte coletivo.

O cenário retratado pela pesquisa está relacionado à política do poder público de descentralização habitacional no período de evolução urbana de Cuiabá, levando a população de baixa renda para regiões mais afastadas (VASCONCELOS; MARINETE, 2009). Culminando no aumento das distâncias a serem percorridas, o que dificulta e encarece a oferta de transporte coletivo. Dessa forma, a população de renda mais baixa tem a sua mobilidade limitada e, logo, menor acessibilidade.

\section{CONCLUSÕES}

A UFMT se caracteriza como um PGV, visto que atrai um grande número de viagens coincidentes com os picos de tráfego no sistema viário e oriundas de várias regiões do munícipio de Cuiabá. Pela análise de divisão modal, averiguou-se que o transporte individual motorizado é o principal meio utilizado para acessar o campus, seguido pelo transporte público e transporte ativo. Esse último, por sua vez, poderia ser mais aproveitado com a implantação infraestrutura urbana, estimulando o maior uso desse meio pelas zonas localizadas em torno do campus.

A partir da análise dos padrões de viagens destinada a universidade, evidencia-se uma possível relação entre a distância média e o modo de transporte, refletindo diretamente no percentual de viagens nos modos de transportes motorizados. É possível observar a tendência de adoção do veículo individual em áreas de renda mais elevada. Adicionalmente, quanto menor a renda, maior é a tendência de utilização de ônibus. Atrelado a esse cenário, é verificado que pessoas de renda familiar baixa habitam em zonas com maior distância média, de $6,88 \mathrm{~km}$, da UFMT. Ademais, somando estes fatores, renda e distância, ao uso e ocupação do solo como também a distribuição espacial das atividades e residências, nota-se a influência desses aspectos na escolha de um meio de transporte em detrimento de outro modo.

Portanto, esse trabalho evidencia o problema do espraiamento urbano ligado a falta de planejamento de uso e ocupação do solo, o que colabora com a desigualdade social e de acesso a população, o que vem contra os princípios da Política Nacional de Mobilidade Urbana, Lei oㅜ 12.587 de 2012, que busca uma gestão democrática e equidade no acesso dos cidadãos ao transporte público coletivo e no uso do espaço público.

\section{REFERÊNCIAS}

ALVES, R. et al. Instituições de ensino como polos geradores de viagem: as diferenças espaciais e temporais nos padrões de viagens. Anais... ANPET, Ouro Preto, p.2632-2643, 13 nov. 2015.

ARAÚJO, M. R. M. de et al. Transporte público coletivo: discutindo acessibilidade, mobilidade e qualidade de vida.: discutindo acessibilidade, mobilidade e qualidade de vida. Psicologia \& Sociedade, [s.l.], v. 23, n. 3, p. 574-582, dez. 2011. Fap UNIFESP (SciELO). http://dx.doi.org/10.1590/s0102-71822011000300015.

BALTAR, M. Perfil da utilização do transporte no campus de Cuiabá da Universidade Federal de Mato Grosso. Trabalho de Graduação. Universidade Federal de Mato Grosso, 2010

BELUFI, F. N.et al. Análise dos padrões de viagens de um polo gerador de viagens, aplicado ao campus universitário da UFMT, em Cuiabá. Anais... II Simpósio Nacional de Gestão e Engenharia Urbana SINGEURB 2019, São Paulo... Anais: Porto Alegre: ANTAC, 2019. 
BENDER, J. É. C.; BALTAR, M. L. de B. Perfil de deslocamentos dos usuários de um Campus Universitário urbano, caso da UFMT em Cuiabá. Anais... Arena ANTP, 2019, São Paulo.

GOMIDES, A. de A. Mobilidade urbana, iniquidade e políticas sociais. Políticas Sociais: acompanhamento e análise, Brasília, n. 12, p. 242-250, 2006.

MEDEIROS, M. G. et al. Transporte ativo em um campus universitário urbano: caso da UFMT. Anais... II Simpósio Nacional de Gestão e Engenharia Urbana: SINGEURB, São Paulo. Anais... Porto Alegre: ANTAC, 2019.

NADALIN, V.; IGLIORI, D. (2015) Espraiamento urbano e periferização da pobreza na região metropolitana de São Paulo: evidências empíricas. Revista EURE, v.41 (124), Santiago.

PORTUGAL, L. da S.; GOLDNER, L. G. Estudos de Polos Geradores de Tráfego e seus Impactos nos Sistemas Viários e de Transportes. 1. ed. São Paulo: Editora Edgard Blucher, 2003. 332 p.

QGIS.org, 2021. QGIS Geographic Information System. QGIS Association. http://www.qgis.org

REDPGV - REDE DE PÓLOS GERADORES DE VIAGENS. O que é um pgv. Disponível em: $<$ http://redpgv.coppe.ufrj.br/index.php/pt-BR/conceitos/o-que-e-um-pgv>. Acesso em: 20 ago. 2019.

SOUZA, S. C. F. de. Modelos para estimativa de viagens geradas por instituições de ensino superior. 2007. 181 f. Dissertação (Mestrado) - Curso de Engenharia Civil, Departamento de Engenharia Civil e Ambiental, Universidade de Brasília, Brasília, 2007.

UNIVERSIDADE FEDERAL DE MATO GROSSO. Anuário Estatístico 2019: Ano base 2018. Cuiabá: UFMT, $2019 . \quad 140 \quad$ p. $\quad$ Disponível em: $<$ https://www1.ufmt.br/anuarioestatistico/arquivos/8e272a000256398cbbe2726e67154361.pdf>. Acesso 17 nov. 2019

VASCONCELOS, L. C. da S.; COVEZZI, M. O Processo de Expansão Urbana de Cuiabá - Mato Grosso, Brasil. In: XII Encuentro de Geógrafos de América Latina, 2009, Montevideo. In: XII Encuentro de Geógrafos de América Latina. Montevideo: Universidad de la República, 2009. v. 1. 